\title{
Evitación experiencial, rumiación e impulsividad en estudiantes de la Universidad Nacional Mayor de San Marcos
}

\author{
Experiential avoidance, rumination and impulsivity in students of the \\ Universidad Nacional Mayor de San Marcos
}

\author{
Carlos Velásquez Centeno ${ }^{* 1}$ \\ Alex Grajeda Montalvo ${ }^{2}$ \\ Víctor Montero López ${ }^{3}$ \\ William Montgomery Urday ${ }^{4}$ \\ Kevin Egusquiza Vásquez ${ }^{5}$ \\ Universidad Nacional Mayor de San Marcos
}

Recibido: $19-04-18$

Aceptado: $23-07-18$

\section{Resumen}

La presente investigación tiene por objeto evaluar la relación entre la evitación experiencial, la rumiación y la impulsividad en los estudiantes de la Universidad Nacional Mayor de San Marcos, en función a la edad, el sexo y al área profesional. Los instrumentos utilizados fueron el Cuestionario de Aceptación y Acción (AAQ-II), la Escala de Impulsividad de Barratt (BIS-11) y la Escala de Respuestas Rumiativas (RRS). La aplicación de los instrumentos se llevó a cabo en el ambiente natural de los estudiantes. El diseño fue asociativo y correlacional. Los resultados indican correlaciones directas, significativas y mayormente moderadas entre evitación experiencial y rumiación, evitación experiencial e impulsividad; y entre rumiación e impulsividad.

Palabras clave: Psicología clínica; análisis conductual; evitación experiencial; impulsividad; rumiación.

\footnotetext{
1 Docente de la Universidad Nacional Mayor de San Marcos. Lima, Perú.

2 Docente de la Universidad Nacional Mayor de San Marcos. Lima, Perú. Email: agrajedam@unmsm.edu.pe 3 Docente de la Universidad Nacional Mayor de San Marcos. Lima, Perú. Email: vmonterol@unmsm.edu.pe 4 Docente de la Universidad Nacional Mayor de San Marcos. Lima, Perú. Email: wmontgomeryu@unmsm.edu.pe, avidolector@yahoo.es

5 Bachiller en Psicología por la Universidad Nacional Mayor de San Marcos. Lima, Perú. Email: kev9nhev@ gmail.com

*Autor para correspondencia. Email: cvelasquezc@unmsm.edu.pe

(C) Los autores. Este artículo es publicado por la Revista de Investigación en Psicología de la Facultad de Psicología, Universidad Nacional Mayor de San Marcos. Este es un artículo de acceso abierto, distribuido bajo los términos de la licencia Creative Commons Atribucion - No Comercia_Compartir Igual 4.0 Internacional. (http://creativecommons.org/licenses/by-nc-sa/4.0/) que permite el uso no comercial, distribución y reproducción en cualquier medio, siempre que la obra original sea debidamente citada.
} 


\begin{abstract}
The purpose of this research is evaluate the relationship between experiential avoidance, rumination and impulsivity in the students of the National University of San Marcos, according to age, sex and professional area. The instruments used were the Acceptance and Action Questionnaire (AAQ-II), the Barratt Impulsivity Scale (BIS-11) and the Ruminative Response Scale (RRS). The application of the instruments was carried out in the natural environment of the students. The design was associative and correlational. The results indicate direct, significant and mostly moderate correlations between experiential avoidance and rumination, experiential avoidance and impulsivity, and between rumination and impulsivity. Keywords: Clinical psychology; behavioral analysis; experiential avoidance; impulsivity; rumination.
\end{abstract}

El desarrollo contemporáneo del análisis conductual en la psicología clínica se ve improntado por una influyente variedad postskinneriana de conductismo, cuyo sustento filosófico se ha denominado "contextualismo funcional", una corriente pragmática de pensamiento argumentada a manera de cosmovisión o "metáfora raíz" inspiradora de quehaceres científicos por el filósofo norteamericano S.C. Pepper en 1942, que lo caracteriza como un enfoque holístico de análisis totalizador de interacciones ocurridas en un contexto histórico y situacional (Hayes, Hayes y Reese, 1988/2016; Hayes, Barnes-Holmes y Roche, 2001). En consecuencia, el contextualismo, a su vez, inspiró el surgimiento de un "ejemplar teórico" o conjunto de abstracciones basadas en propiedades de los eventos que son de interés particular del profesional, dándole sentido a aquellos hechos que él valora como dignos de observar y, ocasionalmente, manipular: la teoría del marco relacional o TMR.

La TMR centra su interés en el estudio del lenguaje, la cognición y la emoción, utilizando como unidad de análisis la operante relacional, una clase funcional de respuesta establecida por contingencias de reforzamiento que, aunque incluye aspectos topográficos sujetos a las dimensiones físicas, no se reduce a ellos. Lo de "relacional" implica la propiedad transituacional típica de la conducta humana, posibilitada por repertorios verbales, de "responder a un evento en términos de otro" (Hayes y Quiñones, 2005; Törneke, 2010/2016). Esta característica de la comunicación verbal, propia del comportamiento guiado por reglas, especifica las consecuencias de las acciones humanas en un contexto lingüístico de referencia sociocultural (Skinner, 1969/1979).

Como fruto de esas disquisiciones, el análisis clínico de la conducta contemporánea utiliza herramientas conceptuales y prácticas para analizar "por qué el lenguaje conduce al sufrimiento" (Hayes y Smith, 2005/2013), es decir, cómo es que los contextos verbales constituyen redes relacionales envolventes con funciones aversivas o motivacionalmente inadecuadas que conducen a trastornos psicológicos. Por lo tanto, la meta de un tratamiento psicoterapéutico eficaz sería la evaluación de tales contextos y su desmantelamiento; algo contrario a la tradicional 
concepción del modelo biomédico, centrado en la población psicopatológicamente afectada y en la topografía de los comportamientos para clasificar sus trastornos psicológicos.

Esto llevó al afán por crear nuevas formas de contacto terapeuta-cliente válidas para toda la población, en los cuales se abordaran los problemas del sufrimiento crónico y los intentos paradójicamente perjudiciales del individuo por evitar su malestar en distintos contextos vitales; elaborándose los procedimientos en "terapia de aceptación y compromiso" (ACT) (Hayes, Strosahl y Wilson, 2012/2014), desde cuyo marco se propone la evitación experiencial como un constructo transdiagnóstico a una gran diversidad de problemas humanos.

La evitación experiencial se concibe como la tendencia del individuo a tratar de controlar, evitar o modificar sus eventos privados — sean éstos pensamientos, sentimientos o recuerdos-, al no estar dispuesto a permanecer en contacto con experiencias percibidas por él como aversivas; a pesar de que a la larga tal pauta de comportamiento llega a ser aún más perjudicial para esa persona que los propios eventos privados indeseables. Se ha planteado una escala para medir este constructo, titulada Acceptance and Action Quiestionnarie (AAQ) (Hayes, Strosahl, Wilson, Bissett, Pistorello, Toarmino, Polusny, Dykstra, Batten, Bergan, Stewart, Zvolensky, Eifert, Bond, Fosyth, Karekla y McCurry, 2004); traducida inicialmente al español europeo por Barraca (2004), y más recientemente modificada por Ruiz, Langer Herrera, Luciano, Cangas y Beltrán (2013). Una versión latinoamericana es la de Patrón-Espinosa (2010).

Desde la perspectiva presente, se considera importante confrontar el constructo de evitación experiencial con otros factores también relacionados con inadecuados contextos motivacionales y verbales, tales como la impulsividad y la rumiación. La impulsividad, por su parte, es definida como una tendencia para actuar veloz e inesperadamente sin tomar en cuenta los efectos perjudiciales para los demás o para uno mismo (Moeller, Barratt, Dougherty, Schimtz y Swann, 2011). La rumiación es el estilo de respuesta por el cual las personas se centran en las sensaciones de tristeza, sus causas o consecuencias, en lugar de plantearse estrategias de resolución de problemas que ayudarían efectivamente a reducir tales eventos privados (Nolen-Hoeksema, 1991). Pérez-Álvarez (2014) agrega que la rumiación es la conducta de analizar repetidamente sobre un mismo hecho, sin que estos sean esclarecedores de la situación, sino más bien como una pauta de evitación.

Existen muchos estudios relacionales sobre evitación experiencial y rumiación, junto con depresión y ansiedad en poblaciones no clínicas, como en el caso de los estudiantes universitarios, en cuyos resultados se muestran relaciones significantes entre las dos variables (Cribb, Moulds, y Carter, 2006; Moulds, Kandris, Starr, y Wong, 2006). Así, Berghoff, Pomerantz, Pettibone, 
Segrist y Bedwell (2012) evaluaron la relación entre la evitación experiencial y la impulsividad en estudiantes universitarios, encontrando una relación significante, siendo la impulsividad de tipo no planeada como la mejor predictora de evitación experiencial. Asimismo Vences (2016) encuentra relación baja, significativa y positiva de 0.32 al estudiar ambas variables en estudiantes escolares y de bachillerato de León y Madrid, cuyas edades fluctuaban entre 13 y 19 años.

Por otra parte, Nicolai (2015) exploró la rumiación e impulsividad como variables mediadoras de las conductas de autolesiones no suicidas y la afectividad negativa en estudiantes universitarios. En su estudio encontró que ambas variables actuaban como mediadoras entre las conductas de autolesiones no suicidas y la afectividad negativa. Así, aquellos con alta afectividad negativa y alta rumiación, o aquellos con alta afectividad negativa y alta impulsividad, tuvieron mayor probabilidad de presentar conductas de autolesiones no suicidas.

La evaluación de la rumiación se ha hecho mediante la escala de NolenHoeksema y Morrow (1991), Ruminative Responses Scale, traducida al español por Hervas (2008). En cuanto a la impulsividad, lo usual ha sido utilizar la conocida escala de Barratt (1959). Barratt Impulsiveness Scale (BIS-11), esta vez recientemente adaptada por Salvo y Castro (2013) al ámbito latinoamericano.

Hervas (2008) adapta al castellano la Escala de respuestas rumiativas. Esta respuesta rumiativo, se concibe como un patrón responsivo en el que hay un exceso de focalización en las variables sintomáticas depresivas. Este estilo se asocia altamente con el desarrollo sintomático depresivo y ansioso. Se eavalaron la vaidez y confiabilidad de la prueba, detectándose dos factores, reflexión y reproches, al igual que en anteriores estudios. Tambien se comprobó una adecuada confiabilidad a través del retest, siendo la consistencia interna mayor que en la prueba original.

Salvo y Castro (2013) describen la fiabilidad y utilidad del La Escala de Impulsividad de Barratt BIS-11. Los autores trabajaron con estudiantes secundarios $(\mathrm{n}=763)$. Se realizaron análisis de consistencia interna y homogeneidad de los ítems para determinar la fiabilidad, y el coeficiente de correlación de Pearson y la prueba t de Student para muestra independiente en el estudio de la validez, obteniéndose un alfa de 0.77 , y correlaciones significativas entre los factores y la impulsividad, lo que indica adecuada validez.

Patrón (2010) traduce y adapta el Acceptance and Action Questionaire AAQII en pobladores de Yucatan, siendo similar con la original. Se modifico el orden de los ítems 1 al 10, mas no se cambió el contenido. Para estudiar las propiedades psicométricas, fue segmentada la muestra, en: con trastornos del estado de ánimo, de ansiedad y psicóticos; y no clínica. La versión mostró ser confiable y válida. Al analizar las diferencias de medias y realizar el análisis de varianza simple se hallaron diferencias significativas entre los no clínicos y los demás grupos 
siendo los psicóticos los que mostraron menor nivel de evitación experiencial. No hubieron diferencias significativas entre los diagnosticados con trastornos del estado de ánimo y los ansiosos.

Tal como se ha evidenciado, el estudio de estas variables en la población universitaria se ha dirigido a buscar la asociación existente entre dos de las tres variables planteadas, mas no en cómo interactúan todas a la vez. Por ello, el presente estudio analiza la relación entre las variables de evitación experiencial, rumiación e impulsividad en estudiantes Sanmarquinos en función a la edad, el sexo y las áreas profesionales. En tal sentido, más detalladamente se evalúa la relación entre la evitación experiencial y la rumiación, la relación entre la evitación experiencial y la impulsividad, y la relación entre la rumiación y la impulsividad, verificando sus ratios de manifestación a través de las escalas antes mencionadas; explorando los niveles de salud-psicopatología desde un modelo funcional y transdiagnóstico en los estudiantes, para así contar con información actualizada sobre la evitación experiencial, rumiación e impulsividad y con medidas efectivas sobre ellas.

A un nivel teórico, el presente estudio permitirá entender mejor los procesos que subyacen a la explicación de los problemas psicológicos y, más ampliamente, al funcionamiento humano; así como también las posibles relaciones que puedan existir entre ellos. A un nivel práctico, los hallazgos permitirán lograr una mejor comprensión acerca de los comportamientos impulsivos, los estilos rumiativos y las pautas de evitación que mantienen los estudiantes universitarios. Asimismo, se ampliará el conocimiento de la evitación experiencial como una posible dimensión funcional diagnóstica a ser utilizada.

\section{MÉTODO}

\section{Muestra}

Se utilizó una muestra accidental, conformada por 2859 estudiantes de los cuales 1,477 son del géneros masculino y 1182 al femenino, cuyas edades fluctúan entre 16 a 46 años los cuales corresponden a cinco Áreas Profesionales: Ciencias de la Salud (659), Ciencias Básicas (349), Ingenieras (654), Ciencias Económicas y de la Gestión (245) y Humanidades y Ciencias Jurídicas y Sociales (702) de la Universidad Nacional Mayor de San Marcos matriculados en el año Académico 2017.

\section{Variables e instrumentos:}

Las variables se midieron por medio de los siguientes instrumentos:

- Evitación experiencial, medida a través del Cuestionario de Aceptación y Acción $(A A Q)$ (Espinosa, 2010). 
- Rumiación, medida a través de la Escala de Respuestas Rumiativas $(R R S)$ (Hervás, 2008).

- Impulsividad, medida a través de la Escala de Impulsividad de Barratt (BIS-11) (Salvo y Castro, 2013).

- Las variables socio demográficas fueron la edad, sexo y áreas profesionales.

\section{Procedimiento:}

La administración de los instrumentos a los alumnos se realizó en coordinación con las autoridades y docentes de la Universidad Nacional Mayor de San Marcos, durante días y horas programadas, en los ambientes naturales y de forma voluntaria.

La información fue procesada a través del Programa SPSS (versión 24), utilizando estadística descriptiva para caracterizar la variable de estudio; la estadística inferencial para establecer la diferencias de acuerdo a la normatividad de la curva, y el análisis de correlación mediante el Coeficiente de Correlación de Speaman.

Cada uno de los tres instrumentos fueron adaptados para nuestra realidad, utilizando para la validez el método del Item-test y para la confiabilidad el Alfa de Crombach. El Cuestionario de Aceptación y Acción (AAQ) y la Escalas de Respuestas Rumiativas mantuvieron su estructura original mientas mientras que la Escala de Impulsividad de Barratt (BIS-11) fue modificada (anexo).

\section{RESULTADOS}

Como se puede observar en la Tabla 1 la evitación experiencial y la rumiacion se encuentran correlacionadas significativa y positivamente tanto en adolescentes, jóvenes y adultos, siendo levemente mayor en los adultos. Asimismo notamos correlación significativa y positiva tanto en varones como en mujeres, siendo levemente mayor en los primeros. De la misma manera la correlación es significativa y positivamente en todas las áreas de formación profesional. Nótese que la correlación menor se da en ciencias de básicas y la mayor en humanidades y ciencias de la salud. 
Tabla 1

Relación entre la evitación experiencial y la rumiación en estudiantes de la Universidad Nacional Mayor de San Marcos, en función a la edad

\begin{tabular}{|c|c|c|c|}
\hline & & & RUMIAC \\
\hline \multirow{3}{*}{ ADOLESCENTES } & \multirow{3}{*}{ EVIEXP } & Correlación de Spearman & $.575^{* *}$ \\
\hline & & Sig. (bilateral) & .000 \\
\hline & & $\mathrm{N}$ & 225 \\
\hline \multirow{3}{*}{ JÓVENES } & \multirow{3}{*}{ EVIEXP } & Correlación de Spearman & $.622^{* *}$ \\
\hline & & Sig. (bilateral) & .000 \\
\hline & & $\mathrm{N}$ & 2315 \\
\hline \multirow{3}{*}{ ADULTOS } & \multirow{3}{*}{ EVIEXP } & Correlación de Spearman & $.663^{* *}$ \\
\hline & & Sig. (bilateral) & .000 \\
\hline & & $\mathrm{N}$ & 119 \\
\hline \multirow{3}{*}{ MASCULINO } & \multirow{3}{*}{ EVIEXP } & Correlación de Spearman & $.620^{* * *}$ \\
\hline & & Sig. (bilateral) & .000 \\
\hline & & $\mathrm{N}$ & 1477 \\
\hline \multirow{3}{*}{ FEMENINO } & \multirow{3}{*}{ EVIEXP } & Correlación de Spearman & $.614^{* *}$ \\
\hline & & Sig. (bilateral) & .000 \\
\hline & & $\mathrm{N}$ & 1182 \\
\hline \multirow{3}{*}{ CIENCIAS DE LA SALUD } & \multirow{3}{*}{ EVIEXP } & Correlación de Spearman & $.659^{* *}$ \\
\hline & & Sig. (bilateral) & .000 \\
\hline & & $\mathrm{N}$ & 659 \\
\hline \multirow{3}{*}{ CIENCIAS BASICAS } & \multirow{3}{*}{ EVIEXP } & Correlación de Spearman & $.585^{* *}$ \\
\hline & & Sig. (bilateral) & .000 \\
\hline & & $\mathrm{N}$ & 349 \\
\hline \multirow{3}{*}{ INGENIERIAS } & \multirow{3}{*}{ EVIEXP } & Correlación de Spearman & $.586^{* *}$ \\
\hline & & Sig. (bilateral) & .000 \\
\hline & & $\mathrm{N}$ & 654 \\
\hline \multirow{3}{*}{ CIENCIAS ECONOMICAS Y DE LA GESTION } & \multirow{3}{*}{ EVIEXP } & Correlación de Spearman & $.608^{* *}$ \\
\hline & & Sig. (bilateral) & .000 \\
\hline & & $\mathrm{N}$ & 295 \\
\hline \multirow{3}{*}{$\begin{array}{l}\text { HUMANIDADES Y CIENCIAS JURIDICAS Y } \\
\text { SOCIALES }\end{array}$} & \multirow{3}{*}{ EVIEXP } & Correlación de Spearman & $.633^{* *}$ \\
\hline & & Sig. (bilateral) & .000 \\
\hline & & $\mathrm{N}$ & 702 \\
\hline
\end{tabular}

La Tabla 2 nos muestra que existen correlaciones significativas pero bajas entre la evitación experiencial y la impulsividad en los estudiantes adolescentes de la Universidad Nacional Mayor de San Marcos. Por el contrario es moderada en jóvenes y adultos, siendo levemente mayor en los últimos. También se observa que existen correlaciones significativas y moderadas entre a evitación experiencial y la impulsividad en función al sexo. Finalmente podemos identificar que existen correlaciones significativas bajas y moderadas entre la evitación experiencial y la impulsividad en función al área de formación profesional, siendo un poco mayor en los estudiantes de humanidades y ciencias jurídicas y sociales. 
Tabla 2

Relación entre la evitación experiencial y la impulsividad en los estudiantes de la Universidad Nacional Mayor de San Marcos, en función a la edad el sexo y las áreas profesionales

\begin{tabular}{|c|c|c|c|}
\hline & & & IMPULSIVIDAD \\
\hline \multirow{3}{*}{ ADOLESCENTES } & \multirow{3}{*}{ EVIEXP } & Correlación de Spearman & $.374^{* * *}$ \\
\hline & & Sig. (bilateral) & .000 \\
\hline & & $\mathrm{N}$ & 225 \\
\hline \multirow{3}{*}{ JOVENES } & \multirow{3}{*}{ EVIEXP } & Correlación de Spearman & $.410^{* *}$ \\
\hline & & Sig. (bilateral) & .000 \\
\hline & & $\mathrm{N}$ & 2315 \\
\hline \multirow{3}{*}{ ADULTOS } & \multirow{3}{*}{ EVIEXP } & Correlación de Spearman & $.519^{* *}$ \\
\hline & & Sig. (bilateral) & .000 \\
\hline & & $\mathrm{N}$ & 119 \\
\hline \multirow{3}{*}{ MASCULINO } & \multirow{3}{*}{ EVIEXP } & Correlación de Spearman & $.426^{* *}$ \\
\hline & & Sig. (bilateral) & .000 \\
\hline & & $\mathrm{N}$ & 1477 \\
\hline \multirow{3}{*}{ FEMENINO } & \multirow{3}{*}{ EVIEXP } & Correlación de Spearman & $.416^{* *}$ \\
\hline & & Sig. (bilateral) & .000 \\
\hline & & $\mathrm{N}$ & 1182 \\
\hline \multirow{3}{*}{ CIENCIAS DE LA SALUD } & \multirow{3}{*}{ EVIEXP } & Correlación de Spearman & $.474^{* * *}$ \\
\hline & & Sig. (bilateral) & .000 \\
\hline & & $\mathrm{N}$ & 659 \\
\hline \multirow{3}{*}{ CIENCIAS BASICAS } & \multirow{3}{*}{ EVIEXP } & Correlación de Spearman & $.281^{* *}$ \\
\hline & & Sig. (bilateral) & .000 \\
\hline & & $\mathrm{N}$ & 349 \\
\hline \multirow{3}{*}{ INGENIERIAS } & \multirow{3}{*}{ EVIEXP } & Correlación de Spearman & $.377^{* *}$ \\
\hline & & Sig. (bilateral) & .000 \\
\hline & & $\mathrm{N}$ & 654 \\
\hline \multirow{3}{*}{$\begin{array}{l}\text { CIENCIAS ECONOMICAS Y DE LA } \\
\text { GESTION }\end{array}$} & \multirow{3}{*}{ EVIEXP } & Correlación de Spearman & $.366^{* *}$ \\
\hline & & Sig. (bilateral) & .000 \\
\hline & & $\mathrm{N}$ & 295 \\
\hline \multirow{3}{*}{$\begin{array}{l}\text { HUMANIDADES Y CIENCIAS } \\
\text { JURIDICAS Y SOCIALES }\end{array}$} & \multirow{3}{*}{ EVIEXP } & Correlación de Spearman & $.483^{* *}$ \\
\hline & & Sig. (bilateral) & .000 \\
\hline & & $\mathrm{N}$ & 702 \\
\hline
\end{tabular}

La Tabla 3 nos muestra que existen correlaciones significativas y moderadas entre rumiación e impulsividad en los estudiantes de la Universidad Nacional Mayor de San Marcos en función a la edad, siendo levemente mayor en los adultos. También evidencia que existe relación directa y significativa moderada entre rumiación e impulsividad en función al sexo. Finalmente se demuestra que existe relación directa y significativa entre rumiación e impulsividad en función a las áreas profesionales, siendo levemente mayor en ciencias de la salud. 
Tabla 3

Relación entre la rumiación y la impulsividad en los estudiantes de la Universidad Nacional Mayor de San Marcos, en función a la edad, el sexo y las áreas profesionales

\begin{tabular}{|c|c|c|c|}
\hline & & & IMPULSIVIDAD \\
\hline \multirow{3}{*}{ ADOLESCENTES } & \multirow{3}{*}{ RUMIACION } & Correlación de Spearman & $.500^{* *}$ \\
\hline & & Sig. (bilateral) & .000 \\
\hline & & $\mathrm{N}$ & 225 \\
\hline \multirow{3}{*}{ JOVENES } & \multirow{3}{*}{ RUMIACION } & Correlación de Spearman & $.471^{* *}$ \\
\hline & & Sig. (bilateral) & .000 \\
\hline & & $\mathrm{N}$ & 2315 \\
\hline \multirow{3}{*}{ ADULTOS } & \multirow{3}{*}{ RUMIACION } & Correlación de Spearman & $.594^{* *}$ \\
\hline & & Sig. (bilateral) & .000 \\
\hline & & $\mathrm{N}$ & 119 \\
\hline \multirow{3}{*}{ MASCULINO } & \multirow{3}{*}{ RUMIACION } & Correlación de Spearman & $.486^{* *}$ \\
\hline & & Sig. (bilateral) & .000 \\
\hline & & $\mathrm{N}$ & 1477 \\
\hline \multirow{3}{*}{ FEMENINO } & \multirow{3}{*}{ RUMIACION } & Correlación de Spearman & $.485^{* *}$ \\
\hline & & Sig. (bilateral) & .000 \\
\hline & & $\mathrm{N}$ & 1182 \\
\hline \multirow{3}{*}{ CIENCIAS DE LA SALUD } & \multirow{3}{*}{ RUMIACION } & Correlación de Spearman & $.550^{* *}$ \\
\hline & & Sig. (bilateral) & .000 \\
\hline & & $\mathrm{N}$ & 659 \\
\hline \multirow{3}{*}{ CIENCIAS BASICAS } & \multirow{3}{*}{ RUMIACION } & Correlación de Spearman & $.489^{* *}$ \\
\hline & & Sig. (bilateral) & .000 \\
\hline & & $\mathrm{N}$ & 349 \\
\hline \multirow{3}{*}{ INGENIERIAS } & \multirow{3}{*}{ RUMIACION } & Correlación de Spearman & $.430^{* *}$ \\
\hline & & Sig. (bilateral) & .000 \\
\hline & & $\mathrm{N}$ & 654 \\
\hline \multirow{3}{*}{$\begin{array}{l}\text { CIENCIAS ECONOMICAS Y DE LA } \\
\text { GESTION }\end{array}$} & \multirow{3}{*}{ RUMIACION } & Correlación de Spearman & $.418^{* *}$ \\
\hline & & Sig. (bilateral) & .000 \\
\hline & & $\mathrm{N}$ & 295 \\
\hline \multirow{3}{*}{$\begin{array}{l}\text { HUMANIDADES Y CIENCIAS } \\
\text { JURIDICAS Y SOCIALES }\end{array}$} & \multirow{3}{*}{ RUMIACION } & Correlación de Spearman & $.487^{* *}$ \\
\hline & & Sig. (bilateral) & .000 \\
\hline & & $\mathrm{N}$ & 702 \\
\hline
\end{tabular}

\section{DISCUSIÓN}

La investigación demuestra que existe relación directa y significativa entre evitación experiencial y rumiación en los estudiantes de la Universidad Nacional Mayor de San Marcos en función a la edad, el sexo y las áreas profesionales. Al respecto se ha determinado que con respecto a la edad la relación es moderada, siendo relativamente mayor en los adultos. En relación al sexo los coeficientes hallados son moderados siendo los hombres las que poseen una correlación relativamente mayor. En cuanto a las áreas profesionales se ha determinado que todas poseen un nivel moderado observándose que la correlación menor se da en ciencias básicas y la mayor en ciencias de la salud. Datos similares son los de Cribb, Moulds, y Carter (2006); Moulds, Kandris, Starr, y Wong (2006) 
quienes analizaron la relación entre la evitación experiencial y la rumiación, junto con depresión y ansiedad en estudiantes universitarios hallando relaciones significativas directas.

De otro lado y con respecto a la relación entre la evitación experiencial y la impulsividad en los estudiantes de la Universidad Nacional Mayor de San Marcos, en función a la edad, el sexo y las áreas profesionales, hemos encontrado relaciones directas y significativas bajas y moderadas. Cabe resaltar que se mantiene relativa mayor relación en los adultos, los varones y los estudiantes de humanidades y ciencias jurídicas y sociales. Estos resultados concuerdan con los de Berghoff, Pomerantz, Pettibone, Segrist y Bedwell (2012) y Vences (2016) quienes evaluaron la relación entre la evitación experiencial y la impulsividad en estudiantes universitarios, y, en escolares y estudiantes de bachillerato, respectivamente, encontrando una relación directa y significativa entre ambas.

Finalmente hemos encontrado relaciones directas y significativas entre rumiación e impulsividad en los estudiantes de la Universidad Nacional Mayor de San Marcos, en función a la edad, siendo moderadas para todos los casos. En relación al sexo esta es moderada y relativamente mayor en los varones; y moderada en todas las áreas profesionales. En todos los casos los datos no están indicando que a mayor rumiación se dará mayor impulsividad. En el mismo sentido Nicolai (2015) exploró la rumiación e impulsividad como variables mediadoras de las conductas de autolesiones no suicidas y la afectividad negativa en estudiantes universitarios, encontrando que aquellos con alta afectividad negativa y alta rumiación, o aquellos con alta afectividad negativa y alta impulsividad, tuvieron mayor probabilidad de presentar conductas de autolesiones no suicidas.

\section{CONCLUSIONES}

Hemos encontrado que a los altos puntajes en evitación experiencial se corresponden con altas puntuaciones en rumiación en los estudiantes de la Universidad Nacional Mayor de San Marcos.

Asimismo a mayor evitación experiencial mayor impulsividad y viceversa en los estudiantes de la Universidad Nacional Mayor de San Marcos.

Finalmente podemos concluir que los estudiantes con mayores niveles de rumiación presentan mayor impulsividad y los que presentan menor rumiación presentan igualmente menor impulsividad en la Universidad Nacional Mayor de San Marcos. 


\section{RECOMENDACIONES}

Elaborar programas preventivos con estudiantes de la Universidad Nacional Mayor de San Marcos en las que se tenga en cuenta las variables evitación experiencial, rumiación e impulsividad, de acuerdo a cada profesión.

Individualizar los programas que se elaboren de acuerdo al área y profesión correspondiente.

Validar los programas elaborados tomando en cuenta, principalmente, los aspectos de construcción y predictividad.

\section{REFERENCIAS}

Barraca, J. (2004). Spanish adaptation of the Acceptande and Action Questionnarie (AAQ). International Journal of Psychology and Psychological Therapy, 4(3), 505-515.

Barratt E. (1959). Anxiety and impulsiveness related to psychomotor efficiency. Perceptual and Motor Skills, 9, 191-198.

Berghoff, C. R., Pomerantz, A. M., Pettibone, J. C., Segrist, D. J., \& Bedwell, D. R. (2012). The relationship between experiential avoidance and impulsiveness in a nonclinical sample. Behaviour change, 29(1), 25-35.

Cribb, G., Moulds, M. L., \& Carter, S. (2006). Rumination and Experiential Avoidance in Depression. BehaviourChange, 23(3), 165-176.

Hayes, S. C., Hayes, L. \& Reese, H. W. (1988/2016). Finding the phylosophical core: A review of Stephen C. Pepper's World Hypotheses. In S.C. Hayes (Ed.).The act in context: The canonical papers of Steven C. Hayes (pp. 57-77). New York: Routledge.

Hayes, S. C., Barnes-Holmes, D., \& Roche, B. (Eds.). (2001). Relational Frame Theory: A Post-Skinnerian account of human language and cognition. New York: Plenum Press.

Hayes, S. C. y Quiñones, R. M. (2005). Características de las operantes relacionales. Revista Latinoamericana de Psicología, 37(2), 277-289.

Hayes, S. C. y Smith, S. (2005/2013). Sal de tu mente. Entra en tu vida: La nueva terapia de aceptación y compromiso. Bilbao: Descleé de Brouwer.

Hayes, S.C., Strosahl, K.D., Wilson, K.G., Bissett, R.T., Pistorello, J., Toarmino, D., Polusny, M.A., Dykstra, T.A., Batten, S.V., Bergan, J., Stewart, S.H., Zvo-lensky, M.J., Eifert, G.H., Bond, F.W., Fosyth, J.P., Karekla, M. \& McCurry, S.M. (2004). Measuring experiential avoidance: A preliminary test of a work-ing model. The Psychological Record, 54, 553-578.

Hayes, S. C., Strosahl, K. y Wilson, K. G. (2012/2014). Terapia de aceptación y compromiso. Proceso y práctica del cambio consciente (Mindfulness). Bilbao: Descleé de Brouwer.

Hervás, G. (2008). Adaptación al castellano de un instrumento para evaluar el estilo rumiativo: la escala de respuestas rumiativas. Revista de Psicopatología y Psicología Clínica, 13(2), 111-121. 
Moeller, F., Barratt, E., Dougherty, D., Schimtz, J., \& Swann, A. (2011). Psychiatric aspects of impulsivity. The American Journal of Psychiatry, 158(11), 1783-1793.

Moulds ML, Kandris E, Starr S, Wong AC. (2007). The relationship between rumination, avoidance and depression in a non-clinical sample. Behav Res Ther. 45(2):51-61.

Nicolai, K. A. (2015). Rumination and Impulsivity as Moderators of the Relationship between Negative Affectivity and Nonsuicidal Self-Injury among College Students (Ph.D. Thesis). Retrieved from Dissertation Abstracts International.

Nolen-Hoeksema, S. (1991). Responses to Depression and Their Effects on the Duration of Depressive Episodes. Journal of Abnormal Psychology, 100(4), 569-582.

Nolen-Hoeksema, S. \& Morrow, J. (1991). A prospective study of depression and postraumatic stress symptoms after a natural disaster: The 1989 Loma Prieta earthquake. Journal of Personality and Social Psychology, 61, 115-121.

Patrón-Espinosa, F. (2010). La evitación experiencial y su medición por medio del AAQII. Enseñanza e Investigación en Psicología, 15(1), 5-19.

Pérez-Álvarez, M. (2014). Las terapias de tercera generación como terapias contextuales. Madrid: Síntesis.

Ruiz, F. J., Langer, A. I., Luciano, C., Cangas, A. J. y Beltrán, I. (2013). Measuring experiential avoidance and psychological in flexibility: The Spanish version of the Acceptance and Action Questionnaire - II. Psicothema, 25(1), 123-129.

Salvo, L. y Castro, A. (2013). Confiabilidad y validez de la escala de impulsividad de Barratt (BIS-11) en adolescentes. Revista Chilena de Neuro-psiquiatría, 51(4), 245-254.

Skinner, B. F. (1969/1979). Contingencias de reforzamiento: Un análisis teórico. México: Trillas.

Törneke, N. (2010/2016). Aprendiendo TMR: Una introducción a la Teoría del Marco Relacional y sus Aplicaciones. España: Didacbook.

Vences, M. (2016). La evitación experiencial en el tabaquismo. Tesis Master de Psicología general sanitaria. Universidad de Oviedo. 